\title{
Avaliação numérica da perda por acomodação de ancoragem na pós-tração
}

ANGELO RUBENS MIGLIORE JUNIOR - ProfESSOR DoutOR

Centro Universitário da Fundação Educacional de Barretos, Unifeb

\section{RESUMO}

Neste ARTIGo É PROPOSTO UM PROCEDIMENTO NUMÉRICO A SER APLICADO PARA AVALIAÇÃO DA PERDA POR ACOMODAÇÃO DE ANCORAGEM EM VIGAS E EM LAJES USUAIS SUBMETIDAS À PÓS-TRAÇÃO, COM TRAJETÓRIA DE CABOS DE PROTENSÃO DIFERENTES DA SITUAÇÃO TRIVIAL E CLÁSSICA DE PARÁBOLA INICIAL SEGUIDA POR TRECHO HORIZONTAL. A FUNDAMENTAÇÃO TEÓRICA DO PROCEDIMENTO NUMÉRICO É APRESENTADA E UMA FUNÇÃO DE INTERPOLAÇÃO NÃO
LINEAR É PROPOSTA PARA OBTENÇÃO DA POSIÇÃO DE BLOQUEIO. EXEMPLOS DE APLICAÇÃO, COM ORGANIZAÇÃO DE TABELAS SEGUNDO UM ROTEIRO PROPOSTO PRÁTICO TAMBÉM SÃO APRESENTADOS. O PROCEDIMENTO NUMÉRICO É SIMPLES E PODE SER UTILIZADO PARA QUALQUER SITUAÇÃO DE CABO DE PROTENSÃO COM TRAJETÓRIA LONGITUDINAL COMPLEXA, COM DIVERSOS SEGMENTOS PARABÓLICOS E LINEARES EM QUALQUER ORDEM.

Palavras-chave: concreto estrutural, protensão, perda imediata.

\section{INTRODUÇÃO}

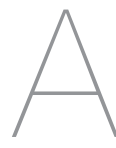

correta avaliação de perdas de protensão é uma letapa importante para o estudo do comportamento de elemento fletido quando submetido a um conjunto de forças de protensão. Parte das perdas imediatas depende da determinação da influência da acomodação ou recuo da ancoragem quando da cravação de cunhas na extremidade de vigas, lajes, bancadas e pistas de protensão.

No sistema construtivo de pré-tração, em geral, não há perda por atrito pela ausência de bainhas, o traçado de cabos de protensão é horizontal e a distância entre cabeceiras de ancoragem é grande para atenuar o efeito de recuo do estiramento inicial do cabo de protensão. O sistema construtivo de pós-tração, em geral, utiliza bainhas metálicas ou de polietileno que podem conduzir a elevadas perdas imediatas de protensão, em razão da complexidade do perfil longitudinal do cabo de protensão acompanhando o andamento da solicitação de flexão simples em peças longas. Nestas condições, a importância da perda por acomodação da ancoragem quando da transferência da força de protensão é amplificada pela trajetória do cabo de protensão, composta por segmentos lineares e parabólicos, tanto no plano vertical quanto no plano horizontal, em razão do aumento de perdas por atrito entre cabo e bainha.

Na prática profissional, a avaliação de perdas imediatas de protensão é composta por três parcelas obtidas em etapas, a saber: a) por atrito; b) por acomodação da ancora- gem; e c) por encurtamento imediato do concreto. Apesar da ocorrência simultânea dos três efeitos físicos, essas etapas são tratadas como independentes e sequenciais, por conduzirem a resultados adequados em obras usuais. A avaliação sequencial das perdas imediatas pode ser constatada pela confrontação direta entre os valores previstos teoricamente e os valores observados em campo do alongamento do cabo de protensão e da contraflecha, desde que tais efeitos sejam obtidos corretamente.

No caso da avaliação da perda por acomodação da ancoragem, Pfeil (1980), Schimd (1998), Pellegrino Neto e Cardoso (2015) e Cholfe e Bonilha (2018) reproduzem expressões analíticas para situações particulares, com trajetória do cabo de protensão composta por um trecho 
inicial parabólico e, eventualmente, com a presença de outro trecho horizontal. Na prática, tais expressões são úteis apenas para estimativa inicial da perda por acomodação em razão da desconsideração do traçado real utilizado para o perfil do cabo de protensão. Como alternativa às expressões analíticas, Carvalho (2017) sugere avaliar a perda por acomodação mediante uma construção gráfica iterativa considerando o traçado efetivo do cabo e que envolve um processo de aproximações sucessivas até a obtenção da solução mais próxima do problema em análise. No caso de lajes com pós-tração não aderente, Aalami (2014) considera a força de protensão com redução única e média ao longo de todo cabo, em virtude da pequena perda por atrito e da complexidade do perfil longitudinal do cabo.

Este artigo apresenta uma solução numérica para estimativa da perda por acomodação envolvendo uma função de interpolação aplicável a elementos protendidos, tanto curtos quanto longos, e com traçado qualquer do perfil longitudinal de cabo de protensão. A principal vantagem da solução numérica apresentada é a possibilidade de obtenção do resultado desejado de modo simples e rápido para utilização em situações práticas reais.

\section{DESENVOLVIMENTO}

A fundamentação teórica do processo numérico proposto envolve a revisão da avaliação da perda por atrito e da perda por acomodação de ancoragem de cabos estirados com o sistema construtivo de protensão por pós-tração. As mesmas condi- ções podem ser generalizadas para o sistema de pré-tração, com as devidas correções.

Por comodidade, neste artigo é utilizada a nomenclatura de cabo de protensão para descrever um ou vários fios ou cordoalhas de aço para protensão de mesma geometria. De mesma posição vertical e de mesma força inicial, agrupados ou não, e caracterizando uma unidade independente de protensão.

\section{I Características da perda por atrito}

A força de protensão $P_{\text {ini }}$ aplicada pelo atuador hidráulico (macaco de protensão) na extremidade de ancoragem ativa de um cabo apresenta redução ou perda ao longo da trajetória do cabo. A força residual após a perda por atrito $P_{x}$ para determinada posição longitudinal $x$, medida pela projeção da trajetória em planta a partir da extremidade ativa, pode ser obtida pela Eq. 1, na qual é introduzida a perda relativa por atrito com o redutor de força $\gamma_{\text {atr }}$ para obtenção da perda $\Delta \mathrm{P}_{\text {atr }}(\mathrm{x})$.

$[1]$
$P_{x}=P_{i n i}-\Delta P_{a t r}(x)=\left(1-\gamma_{a t r}\right) P_{i n i}$

$O$ redutor $\gamma_{\text {atr }}$ pode ser expresso pela relação teórica da Eq. 2, envolvendo o coeficiente de atrito aparente $\mu$ entre cabo e bainha, segundo a somatória dos desvios angulares $\Sigma \alpha$ e o coeficiente de perda por unidade de comprimento $\mathrm{k}$ relacionado com a ondulação parasita ou não intencional na montagem do cabo ao longo de sua trajetória.

$\gamma_{\text {atr }}=\frac{\Delta P_{a t r}(x)}{P_{\text {ini }}}=\exp [-\mu(\Sigma \alpha+k x)]$

Tipicamente, os valores de $\mu$ e $\mathrm{k}$ são pequenos e podem ser estimados para a situação atual e corrente de pós-tração com cordoalhas aderentes e bainhas metálicas como sendo $\mu=0,2 / \mathrm{rad}$ e $\mathrm{k}=1 \% \mathrm{rad} / \mathrm{m}$, segundo a recomendação de ABNT NBR 6118:2014. Para a situação de pós-tração com cordoalhas não aderentes, o valor de $\mu$ é ainda menor e, para a situação de pré-tração, $\mu=0$. O desvio angular total $\Sigma \alpha$ expresso em radianos depende das curvaturas do cabo de protensão ao longo do perfil longitudinal e, na prática, para vigas isostáticas protendidas e cabos de protensão com trajetória composta por segmentos parabólicos abatidos usuais, a quantidade $\Sigma \alpha$ varia entre 0,05 a 0,35 rad. A Eq. 2 pode ser substituída por expansão de série de Maclaurin e simplificada apenas com o primeiro termo, pois o argumento da função exponencial é pequeno (Eq. 3).

$\gamma_{a t r}=\exp [-\mu(\Sigma \alpha+k x)] \approx 1-\mu(\Sigma \alpha+k x)$

Identificando as extremidades i e j de um segmento de cabo de mesma curvatura (ou seja, com trecho parabólico ou linear) como dois pontos notáveis, a conclusão obtida é que a função que representa a força de protensão $P_{x}$ pode ser assumida como sendo linear entre pontos notáveis consecutivos de um segmento de cabo de comprimento em projeção horizontal c. Com essa simplificação e conhecidas as forças nos pontos notáveis $\mathrm{P}_{\mathrm{i}}$ e $\mathrm{P}_{\mathrm{i}}$, a determinação da força residual após as perdas por atrito é obtida com a Eq. 4, para qualquer posição intermediária $x$ entre dois pontos notáveis em função 
da posição relativa $\xi$, medida ao longo de um segmento de cabo em projeção horizontal, conforme a Eq. 5.

$P_{x}=P_{i}(1-\xi)+P_{j} \xi$

$\xi=\frac{x}{c}$

A Figura $1 \mathrm{~b}$ ilustra $\mathrm{o}$ andamento do gráfico que representa a função $P_{x}$ da força de protensão após as perdas por atrito de uma certa viga ABCD esquematizada na Figura 1a, com ancoragens ativa em A e passiva em $D$ para efeito de identificação das quantidades $P_{x}$, $x$ e $c$ de um segmento de cabo qualquer.

\subsection{Avaliação da perda por acomodação de ancoragem}

Após o término da operação de cravação de cunha metálica na extremidade ativa de um cabo de protensão, ocorre recuo $\omega$ do estiramento do cabo ocasionado pelo escorregamento da cunha no interior da cavidade cônica do bloco de ancoragem. Com o sistema atual de ancoragem individual para cada fio ou cordoalha, esse recuo é estimado como sendo de cerca de $6 \mathrm{~mm}$. Esse recuo também já foi estimado como sendo de cerca de $12 \mathrm{~mm}$ para o sistema original de Freyssinet, o qual utilizava uma única cunha central para um conjunto de até 12 cordoaIhas, como descrito por Pfeil (1980). Uma variante do sistema de ancoragem é a fixação de barras roscadas de aço de protensão, fixadas utilizando porcas e contraporcas e cujo recuo da ancoragem é estimado atualmente como sendo de cerca $3 \mathrm{~mm}$.

O efeito da redução do estiramento do cabo em cada extremidade ativa provoca redução da força de protensão instalada, pois implica redução da deformação $\Delta \varepsilon$ no aço de protensão em função da acomodação $\omega$ e da distância $L$, medida entre as ancoragens nas extremidades de um cabo e avaliada pela Eq. 6.

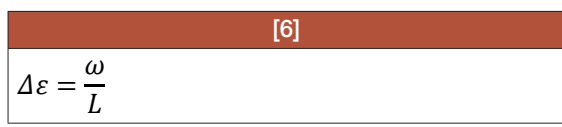

Para o caso de pré-tração, a perda de protensão por acomodação da ancoragem $\Delta \mathrm{P}_{\text {acom }}$ é constante em todo cabo e pode ser avaliada através da aplicação direta da Lei de Hooke (Eq. 7).

[7]

$$
\Delta P_{\text {acom }}=\Delta \sigma_{P} A_{P}=\left(E_{P} \Delta \varepsilon\right) A_{P}=E_{P} A_{P} \frac{\omega}{L}
$$

Onde: $\Delta \sigma_{p}$ é a redução da tensão no aço, $A_{p}$ é a área de aço de um cabo; $\mathrm{E}_{\mathrm{p}}$ é o módulo de elasticidade aparente do aço de protensão.

No caso da pós-tração, a perda de protensão por acomodação também deve considerar a quantidade de energia de deformação total perdida $\Omega$ no trabalho realizado pela força de protensão quando da acomodação $\omega$, pois o cabo em contato com a bainha apresenta perda por atrito, agora em sentido oposto ao atrito devido ao estiramento inicial. Sendo conhecido o recuo $\omega$, a energia de deformação perdida $\Omega$ em certo comprimento é avaliada como sendo dada pela Eq. 8.

$\Omega=E_{P} A_{P} \omega$

A Figura 2a ilustra trecho do gráfico de força de protensão após a perda por acomodação da ancoragem ativa no ponto notável de extremidade A. Admitindo variação linear da força de protensão em segmento de curvatura constante, pode haver posição intermediária S na qual o efeito da perda por atrito durante o recuo do cabo deixa de promover efeitos físicos significativos. A distância s entre a extremidade ativa e essa posição intermediária é normalmente identificada por comprimento de

\section{Figura 1}

a) Esquema de perfil de cabo de protensão de viga com pontos notáveis ABCD; b) Gráfico de força de protensão após perdas por atrito 
bloqueio e interessa conhecer essa posição para avaliar a força residual em todas as posições longitudinais de um cabo.

Como a perda por atrito no comprimento de bloqueio segue variação aproximadamente linear e de mesma declividade daquela correspondente à perda por atrito indicada na simplificação da Eq. 3, a força de protensão $P_{A}$ na extremidade ativa $A$ deve ser reduzida para o valor $P_{A S}$ da quantidade $\Delta \mathrm{P}_{\text {acom }}$, correspondente à perda por acomodação na extremidade A, conforme Eq. 9.

[9]

$P_{A S}=P_{A}-\Delta P_{\text {acom }}$

O triângulo $P_{A}-P_{A S}-P_{S}$ ilustrado na Figura 2a é isósceles e espelhado em relação à força $P_{S}$, em razão da variação linear, e sua área representa a energia total de deformação $\Omega$, a qual é obtida integrando a função de força $P_{x}$ com a posição $x$ no trecho de comprimento de bloqueio $s$ em conformidade com o detalhe da Figura 2b, conforme Eq. 10.

\section{[10]}

$\frac{1}{2} \Omega=\int_{0}^{s} \frac{1}{2}\left(P_{x}-P_{S}\right) d x$

\subsection{Função de interpolação proposta}

Para cada segmento de cabo pode ser admitido que a energia $\Omega$ é obtida pela área formada por retas rebatidas do gráfico de Força - Distância e a posição de bloqueio s está localizada no segmento no qual é atendida a condição expressa na Eq. 11.

[11]

$\Omega_{j-1, j} \geq \Omega$

a)

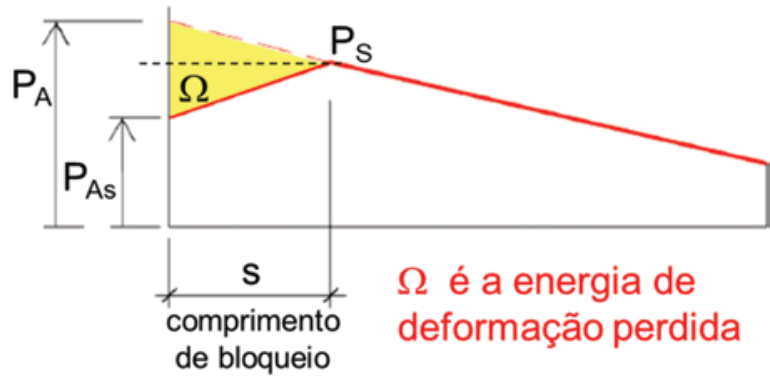

b)

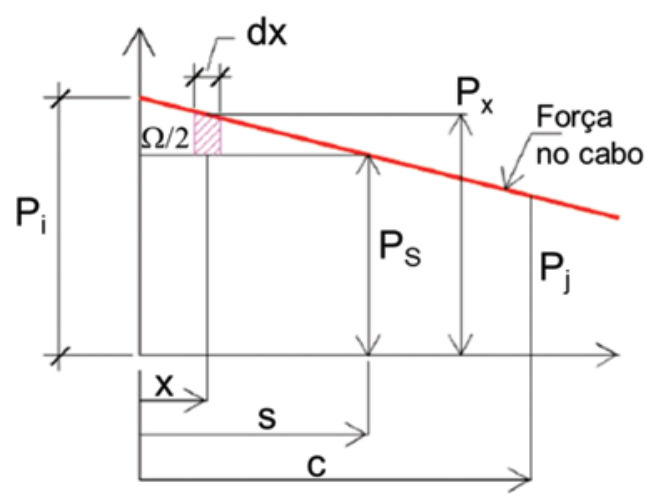

\section{Figura 2}

a) Gráfico de força de protensão após perda por acomodação;

b) Detalhe de integração de força de protensão em posição intermediária

A pesquisa da posição de bloqueio é ilustrada na Figura 3 para a situação de cabo com três segmentos. Caso a área $\Omega_{\mathrm{AB}}$ seja superior à energia $\Omega$, então o bloqueio está no segmento AB. Caso a área $\Omega_{\text {AC }}$ seja superior à energia $\Omega$, então o bloqueio está no segmento $\mathrm{BC}$, e assim sucessivamente.

Conhecido o segmento i que contém a posição de bloqueio, a posição relativa $\xi_{\mathrm{s}}$ pode ser obtida com boa precisão através de uma função de interpolação não linear ilustrada na Figura 4, pois a energia de deformação depende do quadrado das distâncias, segundo Eq. 12.

$\xi_{s}=\frac{s}{c_{i}}=\frac{\sqrt{\Omega}-\sqrt{\Omega_{j-1}}}{\sqrt{\Omega_{j}}-\sqrt{\Omega_{j-1}}}$

Onde: $\Omega_{\mathrm{j}}$ é área do gráfico até a extremidade do segmento considerado e $\Omega_{\mathrm{j}-1}$ é a área do gráfico até a

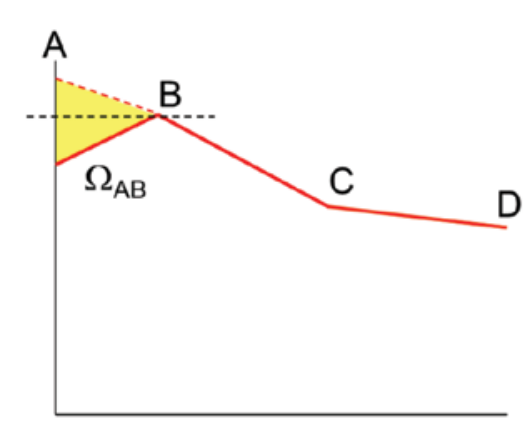

a)

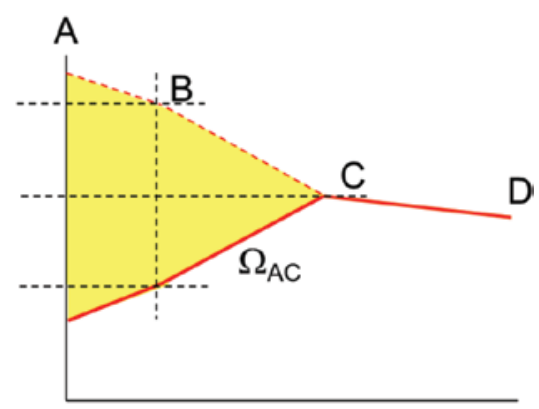

b)
Figura 3

a) Bloqueio no segmento $A B$; b) Bloqueio no segmento $B C$ 


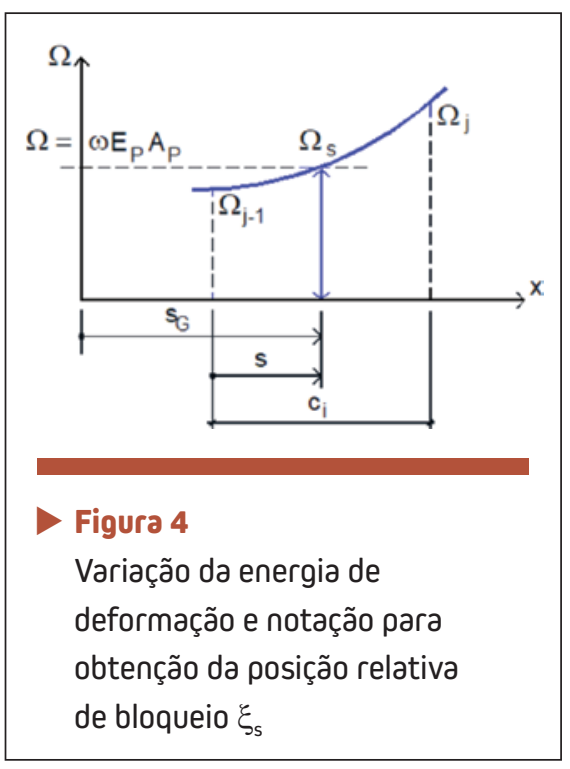

extremidade do segmento anterior. Caso o bloqueio esteja no primeiro segmento, então deve ser imposto $\Omega_{\mathrm{j}-1}=0$. A Eq. (12) coincide numericamente com a expressão analítica clássica de bloqueio no primeiro trecho parabólico ou no segundo trecho horizontal seguinte.

Se é desejável conhecer a posição de bloqueio $s_{G}$ em relação à extremidade ativa, basta totalizar os comprimentos de segmentos anteriores ao bloqueio e somar a distância parcial s. Para casos usuais de cabo curto (pós-tração aderente), de cabo com pouco atrito (pós-tração não aderente) ou de cabo com simetria de ancoragens ativas nas duas extremidades, é possível que a posição de bloqueio não seja encontrada apenas com a Eq. 11. Esse caso é ilustrado na Figura 5 para certo cabo simétrico na posição central C, cuja energia de deformação para ser alcançada exige a área adicional $\Delta \Omega$. A forma geométrica da área adicional no gráfico de força de protensão é a de uma figura composta por segmentos de paralelogramo e é determinada pela Eq. 13.

$[13]$
$\Omega=\Omega_{j}+\Delta \Omega \rightarrow \Delta \Omega=\Omega-\Omega_{j}$

Conhecida a área adicional $\Delta \Omega$, as forças residuais em todos pontos notáveis devem ser reduzidas da quantidade $\Delta \mathrm{P}_{\mathrm{S}}$, independentemente do número de segmentos de cabo em razão do espelhamento do gráfico de força de protensão, segundo Eq. 14.
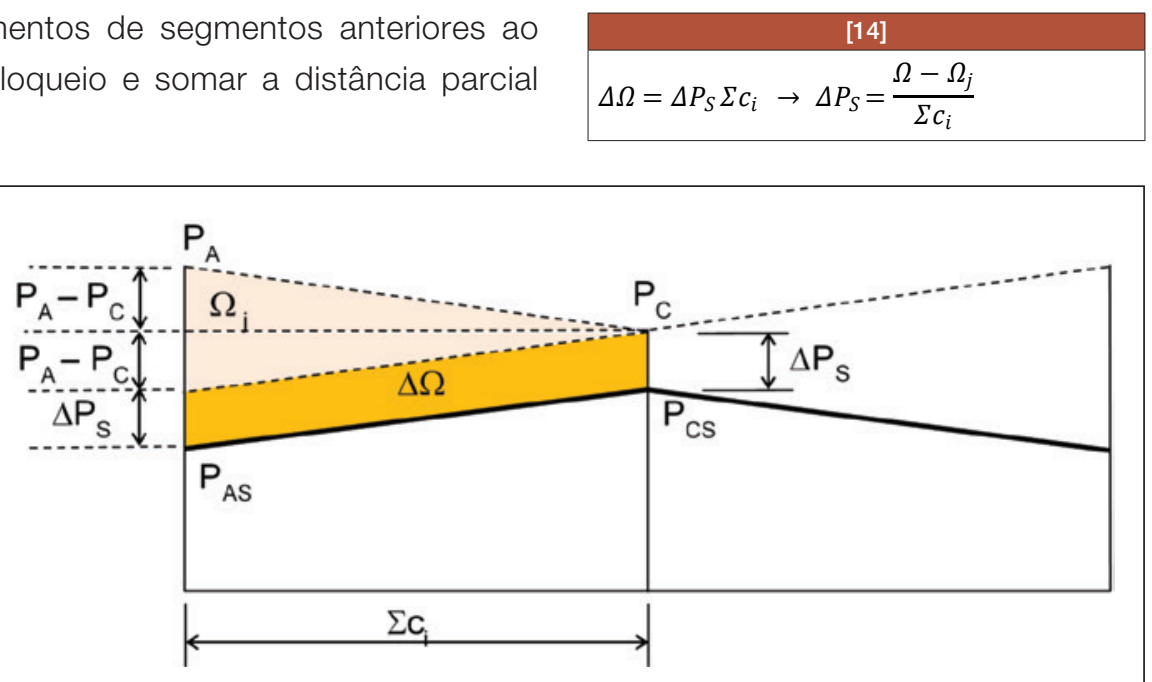

Figura 5

Avaliação da área adicional quando a posição de bloqueio ultrapassa o meio do vão de viga simétrica com cabo curto

\subsection{Procedimento numérico proposto}

Este artigo propõe que a pesquisa da posição de bloqueio devido às perdas por acomodação de ancoragem seja realizada com o seguinte roteiro: a) identificação de pontos notáveis de segmentos de cabo; b) construção de tabela identificando as forças residuais após a perda por atrito nas extremidades de cada segmento; c) construção de tabela identificando a área $\Omega_{\mathrm{j}}$ admitindo que o bloqueio está na extremidade de cada segmento; d) identificação do segmento de cabo que contém a posição de bloqueio; e) identificação de eventual área adicional $\Delta \Omega$ para atendimento da energia de deformação total $\Omega$; f) obtenção da posição relativa de bloqueio $\xi_{\mathrm{s}}$ com a função de interpolação proposta; g) identificação da força de protensão $P_{S}$ na posição de bloqueio; h) redução da força de protensão da quantidade $\Delta \mathrm{P}_{\text {acom }}$ pelo espelhamento do gráfico de força desde a extremidade ativa até a posição de bloqueio; i) eventual redução adicional da força de protensão da quantidade $\Delta \mathrm{P}_{\mathrm{S}}$.

\section{EXEMPLOS DE APLICAÇÃO}

Para ilustração do procedimento numérico, é admitida viga isostática e simétrica com pós-tração aderente, trajetória de cabo de protensão de segmentos $A B C D E$, simétrico em relação ao ponto notável $\mathrm{C}$, com bainha metálica, 15 cordoalhas de aço CP-190 RB (Ep =200 GPa) de diâmetro nominal de $12,7 \mathrm{~mm}$ e área nominal $\mathrm{Ap}=15,15$ $\mathrm{cm}^{2}$ e força de protensão $P_{\text {ini }}=2.000 \mathrm{kN}$ no cabo com extremidades ativas $A$ e E. Impondo que o segmento $A B$ seja 
parabólico de projeção horizontal 20 m e que o segmento BC seja horizontal de comprimento $4 \mathrm{~m}$, pode ser construída a Tabela 1 com as forças de protensão nas extremidades de cada segmento após as perdas por atrito.

\section{I Exemplo I}

Impondo acomodação de ancoragem de cunhas individuais com $\omega=6 \mathrm{~mm}$, a energia de deformação perdida no recuo elástico é dada pela Eq. 15.

\section{[15]}

$\Omega=E_{P} A_{P} \omega=1.818 \mathrm{kN} . \mathrm{m}$

Como é utilizada a hipótese de variação linear da força de protensão em cada segmento, para construção da Tabela 2 a área $\Omega$ pode ser obtida pela Eq. 16.

$[16]$
$\Omega_{j}=2 \sum_{i=1}^{j}\left\{c_{i}\left[\frac{\left(P_{i-1}+P_{i}\right)}{2}-P_{j}\right]\right\}$
$=\sum_{i=1}^{j}\left[c_{i}\left(P_{i-1}+P_{i}-2 P_{j}\right)\right]$

Como observado na última coluna da Tabela 2, o bloqueio está no segmento $A B$, pois é atendida a condição da Eq. 11 e a posição relativa de bloqueio fica determinada com a Eq. (12), a qual é avaliada numericamente pela Eq. 17.

$\xi_{s}=\sqrt{\frac{1.818}{3.443}}=0,727$

Conhecida a posição relativa, a força no cabo no fim da posição de bloqueio é dada pela Eq. 18.

\section{[18]}

$P_{S}=P_{i}\left(1-\xi_{s}\right)+P_{j} \xi_{s}=1.874,9 k N$

A força após a acomodação na posição da ancoragem A é reduzida

Tabela 1 - Força de protensão após perdas por atrito em cada extremidade de segmento

\begin{tabular}{|cccc|} 
Segmento & $c_{i}(m)$ & $P_{i}(k N)$ & $P_{j}(k N)$ \\
$A B$ & 20,0 & $2.000,0$ & $1.827,9$ \\
$C D$ & 4,0 & $1.827,9$ & $1.813,3$ \\
\hline
\end{tabular}

da quantidade $\mathrm{DP}_{\mathrm{A}}$ obtida por meio da Eq. 19.

[19] $P_{A S}=P_{A}-\Delta P_{A}=2 P_{S}-P_{A}=1.749,8 k N$

\subsection{Exemplo 2}

Adotando acomodação de ancoragem $\omega=12 \mathrm{~mm}$, a energia de deformação é o dobro da obtida no Exemplo 1 e resulta em $\Omega=3.636$ kN.m. Comparando com a Tabela 2, a posição de bloqueio está no segmento BC e pode ser avaliada com a Eq. 12 e dada pela Eq. 20.

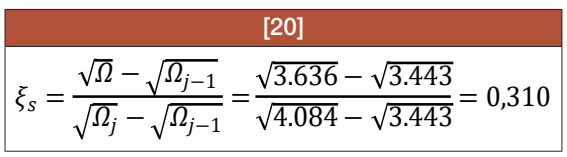

Estimada a posição de bloqueio, a força correspondente a esta posição $S$ fica determinada, bem como as forças nas posições $A$ e $B$, pela Eq. 21.

\begin{tabular}{|l|}
\multicolumn{1}{|c|}{$[21]$} \\
$P_{S}=P_{B}\left(1-\xi_{S}\right)+P_{C} \xi_{S}=1.827,9$ \\
$\times(1-0,310)+1.813,3 \times 0,310=1.823,4 k N$ \\
$P_{A S}=2 P_{S}-P_{A}=2 \times 1.823,4$ \\
$-2.000,0=1.646,7 k N$ \\
$P_{B S}=2 P_{S}-P_{B}=2 \times 1.823,4$ \\
$-1.827,9=1.818,8 k N$ \\
\hline
\end{tabular}

\subsection{Exemplo 3}

Para uma hipotética acomodação de ancoragem $\omega=18 \mathrm{~mm}$, a energia de deformação é o triplo da obtida no Exemplo 1 e resulta em $\Omega=5.454 \mathrm{kN}$.m. Com a Tabela 2, é constatado que $\Omega$ é maior do que $\Omega$ para o segmento BC. Nesse caso particular, a simetria obriga que a posição de bloqueio deve ocorrer na seção central da viga. A solução do problema exige a consideração de área adicional $\Delta \Omega=1.370 \mathrm{kN} . \mathrm{m}$ e a altura do paralelogramo é conhecida com a Eq. 14 e resulta em na Eq. 22.

$\Delta P_{S}=\frac{\Omega-\Omega_{j}}{\sum c_{i}}=\frac{5.454-4.084}{24}=57,1 \mathrm{kN}$

A força no cabo após a acomodação de ancoragem fica reduzida em relação ao Exemplo 2 na seção central e em todas as posições anteriores, conforme Eq. 23.

\section{[23]}

$P_{C S}=P_{C}-\Delta P_{S}=1.813,3-57,1=1.761,3 \mathrm{kN}$

$P_{A S}=2 P_{C}-P_{A}-\Delta P_{S}=2 \times 1.813,3$

$-2.000,0-57,1=1.569,5 \mathrm{kN}$

$P_{B S}=2 P_{C}-P_{B}-\Delta P_{S}=2 \times 1.813,3$

$-1.827,9-57,1=1.741,6 \mathrm{kN}$

Tabela 2 - Área do gráfico de força $\Omega_{\mathrm{i}}$ admitindo bloqueio na extremidade de segmento

$\begin{array}{ccccc}\text { Segmento } & c_{\mathrm{i}}(\mathrm{m}) & \Sigma_{\mathrm{ci}}(\mathrm{m}) & \mathrm{P}_{\mathrm{j}}(\mathrm{kN}) & \Omega_{\mathrm{j}}(\mathrm{kN} \cdot \mathrm{m}) \\ \mathrm{AB} & 20,0 & 20,0 & 1.827,9 & 3.443 \\ \mathrm{CD} & 4,0 & 24,0 & 1.813,3 & 4.084\end{array}$




\section{CONCLUSÕES}

O procedimento numérico proposto é prático e bastante simples de ser aplicado para situações mais complexas do que aquelas indicadas nos Exemplos de Aplicação. De fato, a trajetória de cabos de protensão de vigas e de lajes usuais exige segmento inicial linear e pode haver diversos outros segmentos parabólicos e lineares em qualquer ordem. Para esses casos, a posição de bloqueio não pode ser estimada com precisão adequada com a abordagem analítica clássica.

O caso de lajes com pós-tração não aderente fica facilmente resolvido com o procedimento proposto, sem a necessidade de assumir a simplificação de gráfico de força de protensão com declividade média e constante ao longo do perfil longitudinal.

A função de interpolação proposta para obtenção da posição de bloqueio S intermediária entre dois pontos notáveis consecutivos é simples de ser utilizada, principalmente se houver a confecção de tabelas organizadas segundo os Exemplos de Aplicação.

\section{REFGRÊNCIAS BIBLIOGRÁFICAS}

[1] AALAMI, B. 0. - Post-tensioned buildings: Design and Construction. Palo Alto: Adapt, 2014. International Edition.

[2] ASSOCIAÇÃO BRASILEIRA DE NORNAS TÉCNICAS (ABNT) - NBR 6118:2014: Projeto de estruturas de concreto - Procedimento. Rio de Janeiro: ABNT, 2014, 238p.

[3] CARVALHO, R. C. - Estruturas em concreto protendido. São Paulo: Pini, 2017, 448p.

[4] CHOLFE, L.; BONILHA, L - Concreto Protendido: Teoria e prática. São Paulo: Oficina de Textos, 2018. 350p.

[5] PELLEGRINO NETO, J; CARDOSO, S. D. - Exemplo de uma viga isostática protendida. In: BUENO, S.; KIMURA, A. (coord). ABNT NBR 6118:2014 Comentários e exemplos de aplicação. São Paulo: Ibracon, 2015, 480p.

[6] PFEIL, W. - Concreto protendido. Rio de Janeiro: LTC, 1980, 465p.

[7] SCHIMD, M. T. - Perdas da força de protensão: Publicação técnica 2. Rio de Janeiro: Rudloff, 1998, 17p. Disponível em <http://www.rudloff.com.br/downloads/ publicaca02_perdas_da_forca_de_protensao.pdf $>$ Acesso em 10/08/2020.

\section{Sistemas de Fôrmas para Edifícios}

Recomendações para a melhoria da qualidade e da produtividade com redução de custos

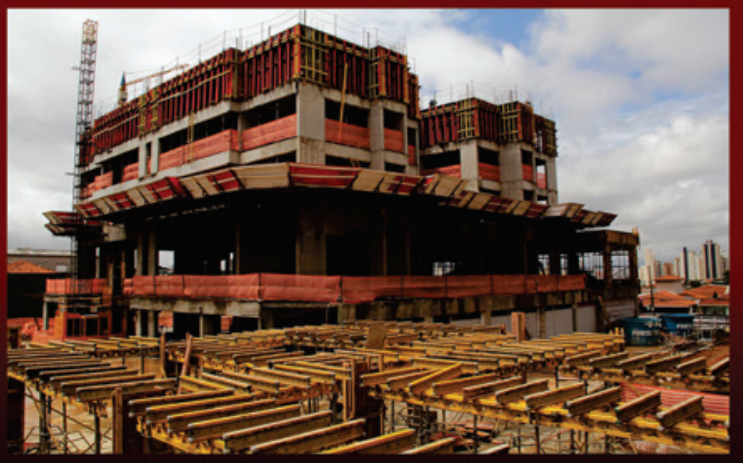

ANTONIO CARLOS ZORZI

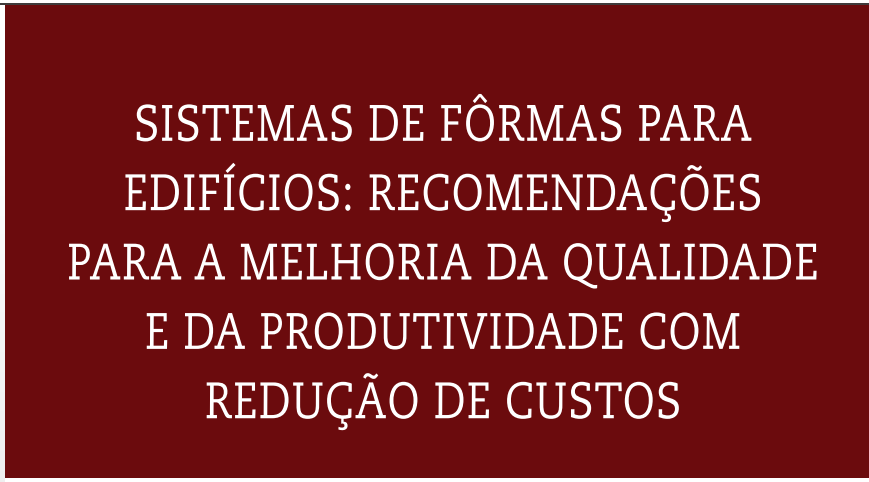

Autor: Antonio Carlos Zorzi

O livro propõe diretrizes para a racionalização de sistemas de fôrmas empregados na execução de estruturas de concreto armado e que utilizam o molde em madeira

As propostas foram embasadas na vasta experiência do autor, diretor de engenharia da Cyrela, sendo retiradas de sua dissertação de mestrado sobre o tema.

\section{DADOS TÉCNICOS}

ISBN 9788598576237

Formato: $18,6 \mathrm{~cm} \times 23,3 \mathrm{~cm}$

Páginas: 195

Acabamento: Capa dura

Ano da publicação: 2015
Patrocínio

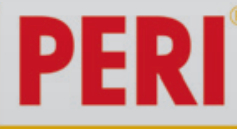

Aquisição: www.ibracon.org.br (Loja Virtual) 